\title{
Atomic scale study of superlow friction between hydrogenated diamond surfaces
}

\author{
S. Dag and S. Ciraci* \\ Department of Physics, Bilkent University, Ankara 06800, Turkey
}

(Received 16 August 2004; published 3 December 2004)

\begin{abstract}
Strong attractive interaction between two clean diamond (001) slabs turns repulsive upon the hydrogenation of surfaces. This repulsive interaction serves as if a boundary lubricant and prevents the sliding surfaces from being closer to each other even under high normal forces. As a result, calculated lateral force variation generated during sliding has small magnitude under high constant loading forces. Superlow friction observed earlier between diamondlike carbon-coated surfaces can be understood by the steady repulsive interaction between sliding surfaces, as well as strong and stiff carbon-carbon and carbon-hydrogen bonds which do not favor energy dissipation. In ambient conditions, the steady repulsive interaction is, however, destroyed by oxygen atoms which chemically modify those stiff surface bonds.
\end{abstract}

DOI: 10.1103/PhysRevB.70.241401

Friction between two surfaces in relative motion is important and a complex phenomenon. ${ }^{1}$ It is induced by short- and long-ranged interaction between surfaces ${ }^{2}$ and may involve phononic, electronic, even photonic energy dissipation, quantum energy transport, structural phase transitions, and various chemical processes. ${ }^{3-10}$ While, for example, construction and tire industries require high friction coefficient, several technological applications aim at very low friction coefficients to achieve low energy dissipation and low wear. Enormous resources are lost through wear and friction. Earlier atomic scale studies have shown that the friction coefficient in the sliding friction is generally lowered as the stiffness of samples increases and the interaction between sliding surfaces decreases. ${ }^{11}$ In boundary lubrication specific foreign atoms or molecules are placed between sliding surfaces to reduce the friction coefficient by cutting down the strong interaction between surfaces. ${ }^{12}$ Recently, Erdemir et al. ${ }^{13}$ reported superlow friction and wear between diamondlike carbon-coated surfaces produced using a hydrogen-rich plasma. They achieved kinetic friction coefficients $\mu_{k}$ as low as 0.001 and wear rates of $10^{-9}-10^{-10} \mathrm{~mm}^{3} / \mathrm{N} \mathrm{m}$ in inert-gas environments under $10 \mathrm{~N}$ load at $0.2-0.5 \mathrm{~m} / \mathrm{s}$ sliding velocities. Hard diamondlike carbon (DLC) films on the moving surfaces have physical properties that are suitable for low friction and wear. ${ }^{14}$ However, observed low magnitude and time-variation of $\mu_{k}$ have a close correlation with the hydrogen content of the source gas.

The work done by Erdemir and his co-workers ${ }^{13}$ has been a breakthrough towards the achievement of superlow friction and long durability in various applications, ranging from the automotive industry to nanotechnology. However, an atomic scale analysis of interactions between sliding surfaces and underlying physical mechanisms leading to superlow friction has been needed to develop coating materials which may be stable in ambient conditions. In fact, the science of friction, i.e., tribology, has made important progress ${ }^{15,16}$ after the discovery of scanning tunneling and atomic force microscopy, allowing atomic resolution of surfaces and the observation of normal and lateral forces of a small fraction of a nanonewton. The character of the tip-surface interaction effects and induced forces has been revealed by first-principles studies. ${ }^{2}$

In this paper we investigated interactions and the resulting physical events between two hydrogenated
PACS number(s): 68.35.Af, 46.55.+d, 68.43. $-\mathrm{h}, 81.40 . \mathrm{Pq}$

diamond(001)- $(2 \times 1)$ surfaces in relative motion by using the first-principles pseudopotential plane-wave method within the density-functional theory. ${ }^{17}$ Hydrogenated DLC (H:DLC) coating is a complex, amorphous structure showing irregularities; their sliding surfaces cannot be commensurate and contain irregularly distributed asperities. The threedimensional structure of the films is important in determining the mechanical properties. ${ }^{18}$ Even if several processes in the sliding friction have a stochastic nature, local bond order and $\mathrm{C}-\mathrm{H}$ bond topology in $\mathrm{H}$ :DLC are similar to various hydrogenated diamond surfaces. Therefore, the interaction between H:DLC surfaces and the resulting friction can be understood by the present model. Here two features, namely the full relaxation of atoms in the surfaces and accurate calculations of lateral force components under the constant loading force $F_{N}$ are of particular importance. Diamond(001)- $(2 \times 1))$ surfaces are represented by two slabs facing each other at a distance, where carbon atoms in the first five surface atomic planes are fully relaxed. To mimic the semi-infinite slab the carbon atoms at the sixth layer and also the $\mathrm{H}$ atoms saturating them are fixed at their equilibrium positions in the optimized bare slab.

Figure 1(a) illustrates two diamond(001)- $(2 \times 1)$ slabs with H-saturated back ends. The structural parameters of the bare surface-forming dimer bonds are successfully reproduced. The contour plots of surface charge density $\rho_{0}$, showing covalent dimer bonds, are presented in Fig. 1(b). The normal force $F_{z}$ originating from the short-range interaction between slabs is calculated after full relaxation of the rest of atoms by minimizing the total energy but keeping the back ends of slabs fixed at a preset distance. A variation of $F_{z}$ is plotted in Fig. 1(c) with respect to the separation between slab surfaces before the relaxation $d_{o}$, as well as the actual separation after the relaxation $d$. While the interaction is weak and repulsive for $d>2.75 \AA, F_{z}$ jumping to contact becomes first strongly attractive and attains the value as high as $\sim-6 \mathrm{eV} / \AA$. Strong bonds form between sliding surfaces when two commensurate slab surfaces are placed at equilibrium separation so that $F_{z} \cong 0$. Once a normal force is applied, $F_{z}$ becomes repulsive since atoms of different surfaces come close to each other at $d<1.5 \AA$. Under these circumstances, $\mu_{k}$ as well as the wear rate are expected to be high in the sliding motion, where sequential bond breaking and re- 
(a)
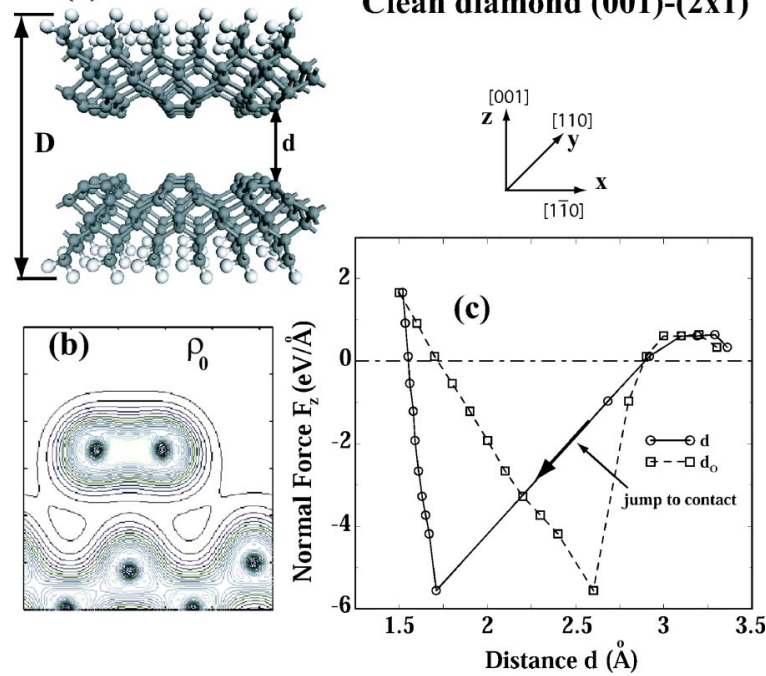

FIG. 1. (a) Two clean diamond(001)- $(2 \times 1)$ surfaces separated by $d$. Back ends of the slabs are saturated by $\mathrm{H}$. The crystal directions are identified by Cartesian axes. (b) The total charge density of the clean diamond(001)- $(2 \times 1)$ surface $\rho_{0}$. (c) A variation of the normal force $F_{z}[\mathrm{eV} / \AA$ per $(2 \times 1)$ cell $]$ as a function of separation $d$ and $d_{o}$. Light and dark balls indicate $\mathrm{H}$ and $\mathrm{C}$ atoms.

bounding take place. The friction constant has been measured to be $\mu_{k}=0.65$ when DLC-coated surfaces are free of hydrogen. ${ }^{19}$

The above situation is, however, dramatically different when all $\mathrm{C}$ dangling bonds on two slab surfaces facing each other are saturated by $\mathrm{H}$ atoms to form a monohydride phase i.e., $\mathrm{H}$ : diamond(001)- $(2 \times 1)$ as depicted in Fig. 2(a). Upon the saturation of surface dangling bonds, a wide energy gap opens between the valence and conduction bands of the slab and the charge density $\rho_{H}$ differs dramatically from $\rho_{0}$ of the clean diamond(001)- $(2 \times 1)$. Moreover, Mulliken analysis indicates 0.25 electrons are transferred from each hydrogen atom to each carbon atom at the surface. This situation is in accord with the fact that $\mathrm{C}$ is more electronegative than $\mathrm{H}$. As a result $\mathrm{H}$ atom is positively charged. At the end, a repulsive short-range force $F_{z}$ is induced between hydrogenated surfaces for $d<2.5 \AA$; the variation of it with the separation $d$ is shown in Fig. 2(c). Although the repulsive force is reduced by the van der Waals attraction between slabs, ${ }^{20}$ it still keeps the sliding surfaces wide apart at a distance $d$ to balances the loading force $F_{N}$. As a result sliding surfaces are prevented from being too close to dissipate energy by deforming $\mathrm{C}-\mathrm{H}$ bonds or by bond breakings. Interestingly, strong attractive interaction between clean $\mathrm{Si}(001)-(2 \times 1)$ surfaces become also repulsive upon the $\mathrm{H}$ saturation of $\mathrm{Si}$ dangling bonds as shown by the inset in Fig. 2(c).

It is important to know whether the repulsive interaction continues to keep surfaces wide apart if one of the diamond slabs are laterally displaced under different loading forces $F_{N}$. To this end, we carried out a series of ab initio total energy $E_{T}$, normal force $F_{z}$, and lateral force $F_{L}$ calculations corresponding to different lateral displacements, $\Delta x$ and $\Delta y$. In these calculations, all the atomic positions are relaxed, except the back end $\mathrm{C}$ and $\mathrm{H}$ layers of both slabs, which are

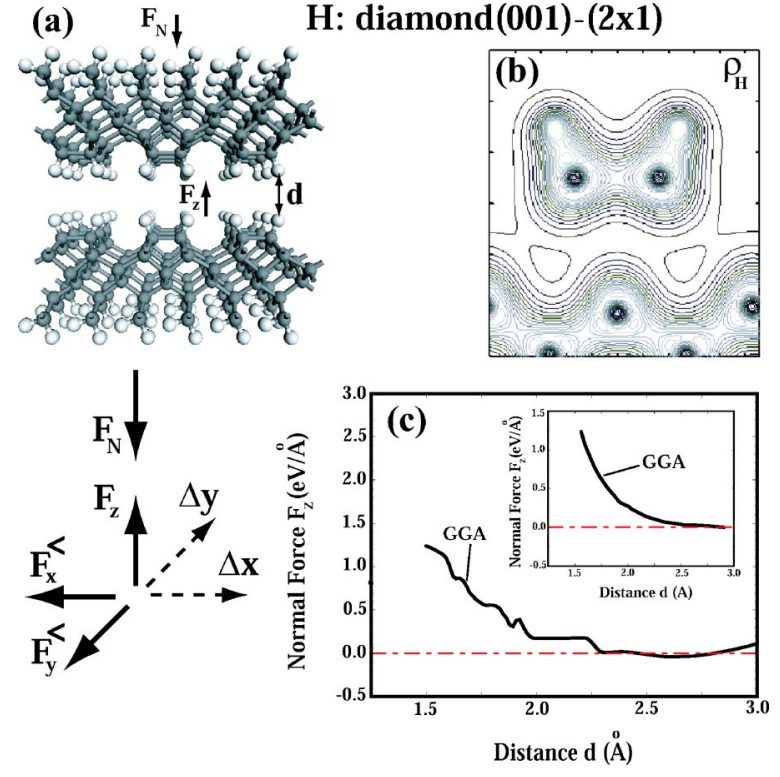

FIG. 2. (a) Two hydrogenated diamond $(001)-(2 \times 1)$ surfaces at a separation $d$. (b) The charge density $\rho_{H}$ of the surface in (a). (c) A variation of the perpendicular, repulsive force $F_{z}$ as a function of actual, relaxed separation between hydrogenated surface $d$. Top and bottom sliding surfaces occupy identical lateral positions. The inset shows the variation of the same force between hydrogenated $\operatorname{Si}(001)-(2 \times 1)$. Lateral displacements $(\Delta x, \Delta y)$, lateral force components $\left(F_{x}, F_{y}\right)$, perpendicular force $F_{z}$, and loading force $F_{N}$ are schematically described.

kept fixed at different distances $D$ to achieve different spacings $d$ between sliding surfaces. Note that keeping two back ends of slabs at a fixed distance $D$ is equivalent to inducing a loading force $F_{N}(D)$, which, in turn, is balanced by $F_{z}$. This way, a database of $E_{T}, F_{z}, F_{L}\left(F_{x}, F_{y}\right)$, and $d$ corresponding to various values of $\Delta x, \Delta y$, and $D$ have been created. The values of $D$ have been varied in small steps to yield normal forces in an appropriate range of interest. In Fig. 3 we present total energy and normal force variations for different lateral displacements $\Delta x$ and $\Delta y$ of the top slab as a function of $d$. We note that $F_{z}$ remains always repulsive and strong even at large $d$. Moreover, for even rather high loading forces the repulsive interaction is able to keep sliding surfaces wide apart, so that $\mathrm{C}-\mathrm{H}$ bonds of different surfaces do not merge. C-H bonds being well separated from each other experience neither wear and rebounding nor a strong deformation during sliding. As a result, dissipation of mechanical energy, and hence $\mu_{k}$, becomes low. Clearly, the generation of a strong repulsive force is the most essential aspect leading to superlow friction and is reminiscent of a boundary lubrication. The variation of $F_{z}(d)$ is not a smooth function owing to the relaxation of $\mathrm{C}-\mathrm{H}$ bonds at different surfaces.

In the course of sliding, the elastic potential energy $V_{E}(\mathbf{r})$ varies periodically. The periods of variation are determined by the lattice parameters of the surface unit cell if the surfaces in relative motion are commensurate as in Figs. 1 and 2. $V_{E}$ has the maximum value when the dimer bonds of sliding surfaces face each other, but it becomes minimum when the upper slab is displaced by a half of the unit cell. During sliding, $V_{E}$ decreases from its maximum to minimum value, 


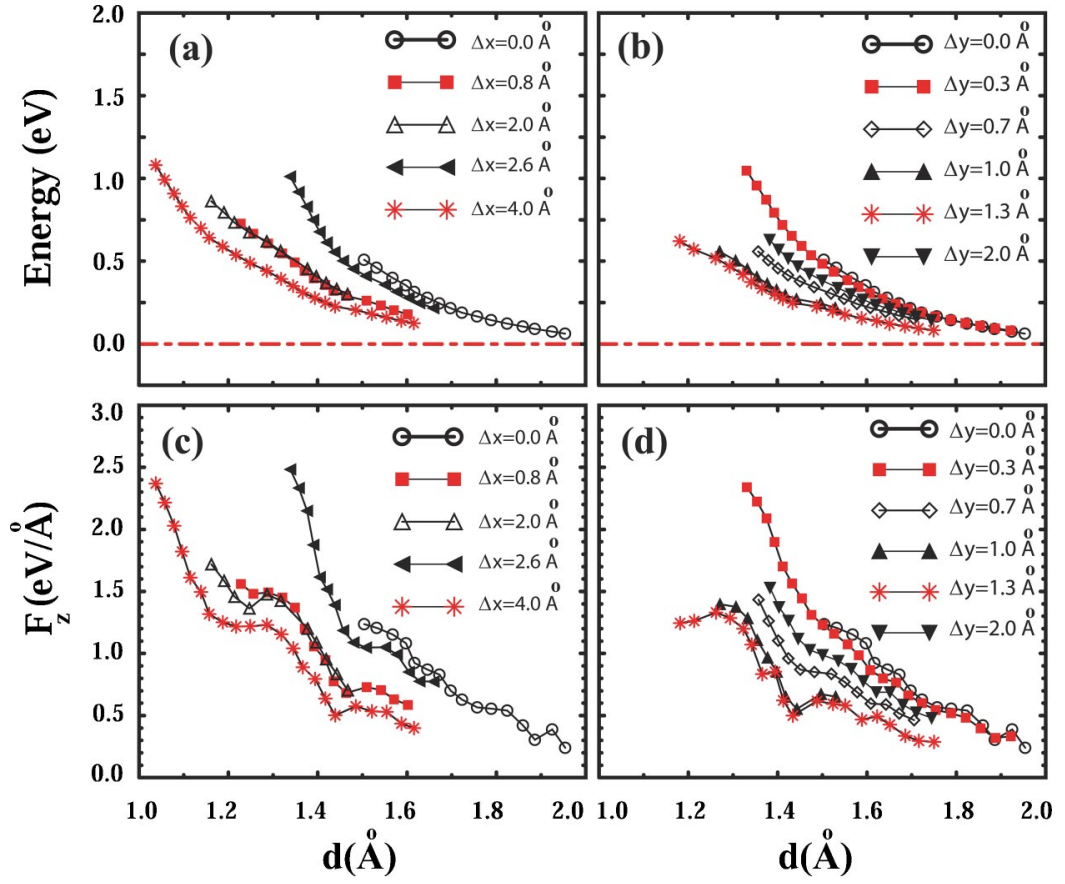

FIG. 3. (Color online) (a) and (b) Variation of total energy $E_{T}$ as a function of perpendicular distance $d$, calculated at different lateral displacements $\Delta x$ and $\Delta y$, respectively. The total energy at very large $d$ is set equal to zero. (c) and (d) The same variation of normal force $F_{z}$. Energy and force units are given for per $(2 \times 1)$ cell. since part of the mechanical energy $\Delta V_{E}$ already spent on the elastic deformation of the slabs, as well as on the bending of $\mathrm{C}-\mathrm{H}$ bonds at the surfaces, is released. As for the lateral force with components $F_{x, y}=-\partial V_{E}(\mathbf{r}) / \partial x, y$, it has also the same period as $V_{E}$. Moreover, it is conservative for fully adiabatic sliding motion: First, it is parallel to the direction of sliding motion, then it becomes antiparallel so that $\int F_{L} d \eta=0$ for full period displacement along $\eta$. However, if sliding motion is not adiabatic but involves sudden changes, part of the stored elastic energy is dissipated by generating nonequilibrium phonon distribution. Once any elastic deformation state is suddenly released, the phonon distribution in thermal equilibrium at $T_{0}, \Psi\left(n_{1}^{0}, n_{2}^{0}, \ldots n_{q}^{0}, \ldots\right)$, changes to nonequilibrium distribution $\Psi\left(n_{1}, n_{2}, \ldots n_{q} \ldots\right)$ with excess phonons with mode frequency $\Omega_{q}, \Delta n_{q}=n_{q}\left(\Omega_{q}\right)-n_{q}^{0}\left(\Omega_{q}\right)$, $q=1,2,3, \ldots, 3 N$. In principle, nonequilibrium distribution $\Psi\left(n_{1}, n_{2}, \ldots, n_{q} \ldots\right)$ can be obtained if a particular deformation state is known. ${ }^{6}$ As a simple example, let us consider a $\mathrm{C}-\mathrm{H}$ bond [with a salient surface phonon stretch mode of $\hbar \Omega_{q} \sim 360 \mathrm{meV}$ and a bending mode of $150 \mathrm{meV}$ (Ref. 21)], which is deformed by $b_{q}$. Then the excess phonon energy to be dissipated after the release of deformation can be estimated from $\delta V_{E}=\Sigma_{q} \frac{1}{2} M \Omega_{q}^{2} b_{q}^{2} \cong \Sigma_{q} \Delta n_{q} \hbar \Omega_{q}$. Our earlier model study using the reduced density matrix approach ${ }^{22}$ has shown that mechanical energy stored in nonequilibrium phonons created by stick-slip motion $\Delta V_{E}$ dissipates with a relaxation time $\tau(T=300 \mathrm{~K}) \sim 1 \mathrm{ps}$, according to the expression $\Delta V_{E}[1-\exp (-t / \tau)]$. Using the scaling arguments similar equation with frequency dependent $\tau\left(\Omega_{q}\right)$ has been obtained for the rate of decay. ${ }^{6}$ The calculation of $\mu_{k}$ using the above microscopic approach, however, requires precise characterization of sliding surfaces.

An upper limit for $\mu_{k}$ can be estimated from a global approach using the variation of $F_{L}$ obtained from present calculations. To this end let us consider displacements along the $x$ and $y$ axes and we construct a lateral force variation under a constant loading force from our database, namely $F_{L=x}\left(\Delta x, \Delta y=0, F_{N}\right)$ and $F_{L=y}\left(\Delta x=0, \Delta y, F_{N}\right)$. Keeping $F_{N}$ constant is the most difficult part of our study and requires a large number calculations corresponding to different $(\Delta x ; \Delta y ; d) . F_{N}=1$ and $1.2 \mathrm{eV} / \AA$ per cell taken for the lateral force variations are actually too high as compared to the loading force in the experiment. ${ }^{13}$ The variation of $F_{x}$ and $F_{y}$ illustrated in Fig. 4 is not smooth because the present modeling of sliding is not fully adiabatic. Since lateral force is calculated using coarse displacement steps, the elastic deformation of slabs and $\mathrm{C}-\mathrm{H}$ bonds induced by sliding can be released suddenly. This is the stick-slip process described by the Tomlinson model. ${ }^{23}$ Of course, error bars involved in the
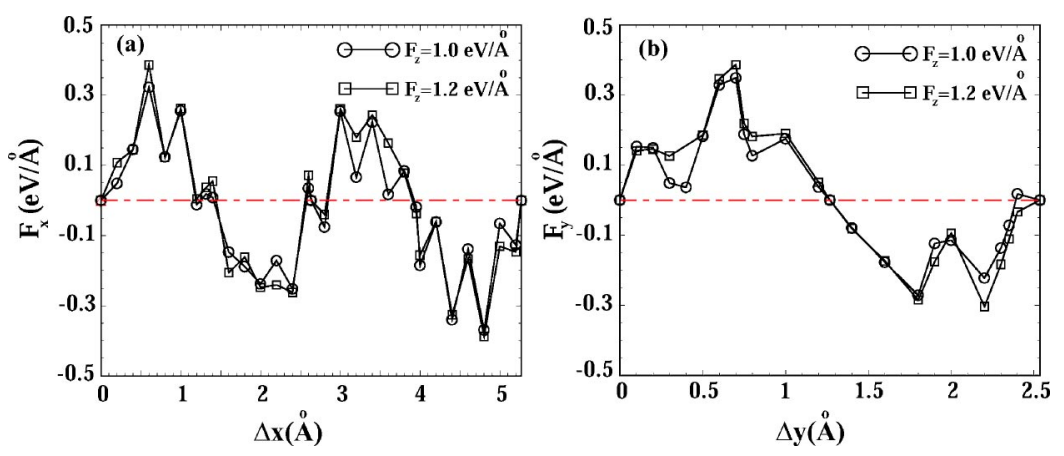

FIG. 4. (a) and (b) Variation of lateral force $F_{x}$ and $F_{y}[$ in $\mathrm{eV} / \AA$ per $(2 \times 1)$ cell] as a function of displacement of the top surfaces $\Delta x$ and $\Delta y$, respectively. Loading force $F_{N}$ is taken constant in the course of sliding. 


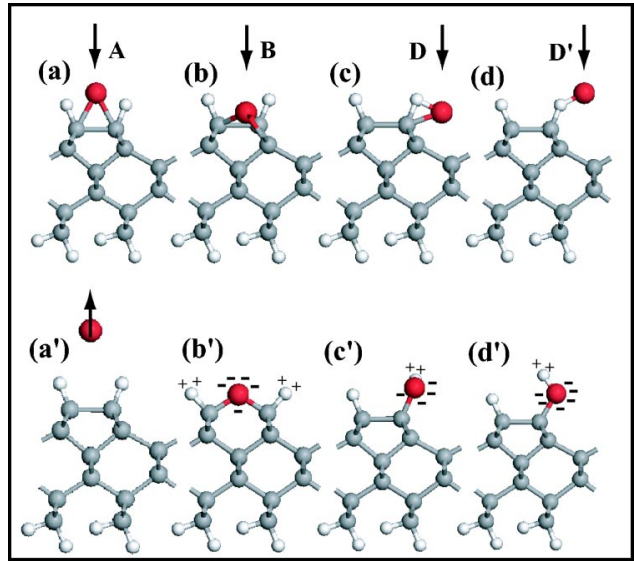

FIG. 5. (Color online) (a)-(d) $\mathrm{O}$ atoms are placed at different location $\left(A, B, D, D^{\prime}\right)$ at the close proximity of the $\mathrm{H}$ : diamond(001)- $(2 \times 1)$ surface before relaxation. $\left(a^{\prime}\right)-\left(d^{\prime}\right)$ Structure and bonding after relaxation.

calculation of forces, as well as in achieving the constant loading-force constraint by a limited number of data can lead to hysteric variation of the lateral force. Now as an ad hoc approach to estimate $\mu_{k}$ in an energy dissipating medium, we assume that the work done by the lateral force $F_{L}$ (i.e., when it is parallel to the direction of motion $F_{L}^{>}$) is totally lost ${ }^{9}$ and we calculate the average friction force $F_{f}$. For displacement along the $x$ axis, $\overline{F_{f}}=\int F_{x}^{>} d x / R$ (here $R$ is a period of motion). Then, the kinetic friction constant is estimated to be $\mu_{k}=\overline{F_{f}} / F_{N}$. Using Fig. 4(a) we found $\overline{F_{f}} \sim 0.05 \mathrm{eV} / \AA$ for $F_{N}=1 \mathrm{eV} / \AA$, and $\overline{F_{f}} \sim 0.07 \mathrm{eV} / \AA$ for $F_{N}=1.2 \mathrm{eV} / \AA$. The corresponding kinetic friction constant is $\mu_{k} \sim 0.05$ for both cases. For the reasons discussed above, a more realistic estimation could be obtained from $\overline{F_{f}}=\int\left(F_{x}^{<}+F_{x}^{>}\right) d x / R$ if lateral force variation were calculated precisely. Our crude force variation in Fig. 4(a) yields $\mu_{k} \sim 0.01$. It is known that in the adiabatic sliding process of commensurate surfaces, $F_{x, y}$ are superposed to yield a high lateral force, but they are conservative. In the case of incommensurate surfaces, the lateral force is relatively lower owing to the cancellations. H:DLC coated surfaces can be viewed as incommensurate except that the disorder gives rise to high-energy dissipation. Our estimation of $\mu_{k}$ obtained from hydrogenated diamond surfaces with the assumption that all mechanical energy stored into elastic energy is dissipated, is an upper limit for $\mathrm{H}$ :DLCcoated surfaces, but it is still too low. Consequently, the physical mechanisms revealed in this study is expected to provide a clear understanding for measured superlow friction. ${ }^{13}$

Finally, we address the issue why H:DLC films can be unstable in atmospheric conditions. The oxygen atom is the potential candidate which destroys the superlow friction when H:DLC coating is exposed to the air. To test we placed $\mathrm{O}$ atoms at different sites of the $\mathrm{H}$ :diamond $(001)-(2 \times 1)$ surface. Upon relaxation $\mathrm{O}$ has been adsorbed. Favorably, it attacked the $\mathrm{C}-\mathrm{H}$ bonds by entering between $\mathrm{C}$ and $\mathrm{H}$ atoms to form $\mathrm{C}-\mathrm{O}-\mathrm{H}$ radicals. Charge transferred to $\mathrm{O}$ from $\mathrm{H}$ and $\mathrm{C}$ makes $\mathrm{O}$ negatively charged as shown in Fig. 5. This way, the steady repulsive interaction between surfaces prior to the oxygenation has ceased to destroy superlow friction.

In conclusion, we modeled the sliding friction between hydrogenated diamond(001)- $(2 \times 1)$ surfaces, and revealed important ingredients leading to superlow friction: These are (i) repulsive interaction between sliding surfaces generated by hydrogenation which persists at any relative position of these surfaces and is strong even at a large distance to prevent $\mathrm{C}-\mathrm{H}$ bonds of disordered surfaces from merging; (ii) strong and stiff $\mathrm{C}-\mathrm{H}$ bonds and a stiff diamond crystal itself prevent excessive energy from dissipation. It is found that oxygenation of surfaces in the atmospheric conditions destroys the steady repulsive interaction.

\footnotetext{
*Electronic address: ciraci@fen.bilkent.edu.tr

${ }^{1}$ B.N.J. Persson, Sliding Friction: Physical Principles and Applications (Springer-Verlag, Berlin, 2000).

${ }^{2}$ S. Ciraci et al., Phys. Rev. B 42, 7618 (1990); 46, 10411 (1992).

${ }^{3}$ J.B. Sokoloff, Phys. Rev. Lett. 71, 3450 (1993).

${ }^{4}$ M. Cieplak et al., Science 265, 1209 (1994).

${ }^{5}$ M.S. Tamassone et al., Phys. Rev. Lett. 79, 4798 (1997).

${ }^{6}$ A. Buldum et al., Phys. Rev. B 59, 16042 (1999); Europhys. Lett. 40, 11969 (1999).

${ }^{7}$ B.N.J. Persson, Phys. Rev. B 44, 3277 (1991).

${ }^{8}$ A. Dayo et al., Phys. Rev. Lett. 80, 1690 (1998).

${ }^{9}$ W. Zhong and D. Tomanek, Phys. Rev. Lett. 64, 3054 (1990); D.

Tomanek et al., Europhys. Lett. 15, 887 (1991).

${ }^{10}$ C. Daly and J. Krim, Phys. Rev. Lett. 76, 803 (1996).

${ }^{11}$ A. Buldum and S. Ciraci, Phys. Rev. B 55, 2606 (1997).

${ }^{12}$ B.N.J. Persson, Phys. Rev. B 55, 8004 (1997).
}

${ }^{13}$ A. Erdemir et al., J. Vac. Sci. Technol. A 18, 1987 (2000).

${ }^{14}$ C. Donnet, Surf. Coat. Technol. 100/101, 180 (1998).

${ }^{15}$ C.M. Mate et al., Phys. Rev. Lett. 59, 1942 (1987).

${ }^{16}$ E. Meyer, R. Overney et al., Phys. Rev. Lett. 69, 1777 (1992).

${ }^{17}$ Calculations have been performed by using the vASP software: G. Kresse and J. Hafner, Phys. Rev. B 47, 558 (1993); G. Kresse and J. Furthmüller, ibid. 54, 11169 (1996).

${ }^{18}$ G.T. Gao et al., J. Phys. Chem. B 107, 11082 (2003).

${ }^{19}$ A. Erdemir, Surf. Coat. Technol. 146, 292 (2001).

${ }^{20}$ For small $d$ the repulsive interaction can be reduced significantly by a van der Waals attractive force. In this range of separation Lifshitz's asymptotic expression is unfortunately not applicable. See Ref. 2

${ }^{21}$ B.D. Thoms and J.E. Butler, Phys. Rev. B 50, 17450 (1994).

${ }^{22}$ A. Ozpineci et al., Phys. Rev. B 62, 10558 (2000).

${ }^{23}$ G.A. Tomlinson, Philos. Mag. 7, 905 (1929). 